\title{
Torsional Potential Energy Surfaces of Dinitrobenzene Isomers
}

\author{
Paul M. Smith and Mario F. Borunda \\ Department of Physics, Oklahoma State University, Stillwater, OK 74078, USA \\ Correspondence should be addressed to Mario F. Borunda; mario.borunda@okstate.edu \\ Received 1 April 2017; Accepted 4 July 2017; Published 20 August 2017 \\ Academic Editor: Gary Wysin
}

Copyright (C) 2017 Paul M. Smith and Mario F. Borunda. This is an open access article distributed under the Creative Commons Attribution License, which permits unrestricted use, distribution, and reproduction in any medium, provided the original work is properly cited.

\begin{abstract}
The torsional potential energy surfaces of 1,2-dinitrobenzene, 1,3-dinitrobenzene, and 1,4-dinitrobenzene were calculated using the B3LYP functional with 6-31G(d) basis sets. Three-dimensional energy surfaces were created, allowing each of the two C-N bonds to rotate through 64 positions. Dinitrobenzene was chosen for the study because each of the three different isomers has widely varying steric hindrances and bond hybridization, which affect the energy of each conformation of the isomers as the nitro functional groups rotate. The accuracy of the method is determined by comparison with previous theoretical and experimental results. The surfaces provide valuable insight into the mechanics of conjugated molecules. The computation of potential energy surfaces has powerful application in modeling molecular structures, making the determination of the lowest energy conformations of complex molecules far more computationally accessible.
\end{abstract}

\section{Introduction}

Torsional potential energy calculations provide conformational information and allow finding the barriers to the rotations of bonds. Early applications were performed in organic compounds phosphates, 1,3-butadiene, polypeptides, and dimethyl groups [1-4]. These initial works showed that if steric interactions are not strong, a rigid rotor model applies. Furthermore, Bongini and Bottoni used density functional theory (DFT) and found that correlation energy effects are to be taken into account for accurate calculations of the conformal properties of organic compounds [4]. The topologies of the PES in the ground state and a selected excited state are useful in understanding the active site of reactions and photoreactivity [1]. PESs were employed to investigate excited-state reaction mechanisms and proton transfer in a complex molecule (2-( $2^{\prime}$-hydroxyphenyl)benzimidazole) and its amino derivatives [2].

The calculations by Bongini and Bottoni compared Hartree-Fock and second-order Møller-Plesset methods [35] to DFT results (at the BLYP and B3LYP levels) $[6,7]$ and determined the structure of the two molecules considered $\left(3,3^{\prime}\right.$-dimethyl-2,2' -bithiophene and $3,4^{\prime}$-dimethyl$2,2^{\prime}$-bithiophene) and to experimental data to quantify the reliability of the models. Both BLYP and B3LYP computations correctly predict both of the rotational minima observed in experiment to an accuracy of $5^{\circ}$. Bongini and Bottoni produced torsional potential energy plots, but their interest was in the nature of the minima, not the characteristics of the energy surface itself [8]. Raman spectra of multiple physical phases of a compound can be used in conjunction with computational ab initio and DFT methods to determine the torsional potential of a two-rotor system and are capable of determining the potential energy surface (PES) with a $3 \%$ accuracy [9]. However, this method requires multiple Raman spectra, several single-point energy computations for representative molecular conformations, and theoretical knowledge of the general form of the PES [9]. The PES of ethoxybenzene, a molecule where the coupling between rotors is small, has been calculated using DFT at the B3LYP level for both a one-rotor molecule and a two-rotor molecule. The points on the PES were computed in $15^{\circ}$ increments and used to construct a torsional PES with a cosine series leastsquares fit to the points and the fit was found to be within $20 \%$ of the experimental values and the two-rotor ethoxybenzene molecule has a planar energy minimum [10].

In this manuscript, we explore the use of the torsional PES to determine the geometry of the lowest energy state 


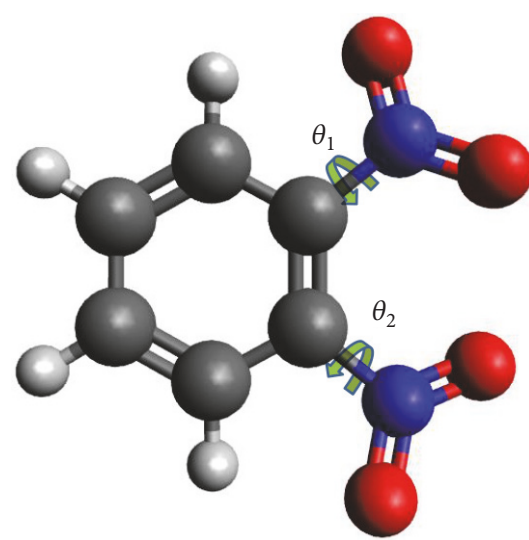

FIGURE 1: Molecular structure of orthodinitrobenzene and definition of the coordinate system. The two-dimensional PESs depend on the angles $\theta_{1}$ and $\theta_{2}$. The orthoisomer has the nitro groups at 1 and 2 positions, the metaisomer has them at 1,3, and the paraisomer has them at 1,4 (the figure was generated with the Avogadro software [11]).

for molecules, a methodology which might be extended to larger molecules such as proteins. We computationally determine the energy correlation between pairs of rotors on the molecule. These correlations can be reduced to a matrix differential equation, and the eigenvalues and eigenvectors of the equation will specify the lowest energy conformation of the molecule being studied. Since the energy of the system is represented by a differential equation in matrix form, it can be iterated computationally to a convergence at the lowest energy state of the molecule. The knowledge of the correlations between different rotors allows local energy minima distinct from the global minima to be easily identified.

We report the torsional PESs for dinitrobenzene isomers. The two-dimensional PES plots show the potential energy of the molecule as a function of the angles each $\mathrm{NO}_{2}$ group makes with respect to the ring. The molecular structure of dinitrobenzene allows for three isomers and thus our calculations explore torsional PESs for modeling conjugated systems with and without steric effects. As shown in Figure 1, the orthoisomer, with the nitro groups at 1 and 2 positions, is characterized by strong steric repulsion. This repulsion is caused by the electrical force between the spatially adjacent oxygen atoms. The 1,3 isomer has attractive intramolecular interactions between the $\mathrm{NO}_{2}$ groups and the $\mathrm{H}$ atoms. The $\mathrm{O}$ atoms have a conjugated negative charge, which is attracted to the conjugated positive charge of the $\mathrm{H}$ ions. Finally, the 1,4 isomer has little steric hindrance or attractive forces affecting the rotation of the $\mathrm{NO}_{2}$ groups. Thus, these three isomers are useful to determine the roles that steric effects and intermolecular attraction play in the rotation of the $\mathrm{NO}_{2}$ groups.

The geometry of the nitro groups in the dinitrobenzene isomers has been explored before. Freed et al., studied the variations in the linewidths of electron spin resonance spectra of the carbon-nitrogen anion for each isomer of the dinitrobenzene anions, where they found alternation in the linewidths of the metaisomer, which arise from rotation of the nitro groups out of the planar conformation [12].
While the alternation was not found in the orthoisomer or paraisomer, the authors posit that the orthoisomer is not similar to the paraisomer because steric interference prevents the orthoisomer from adopting a planar conformation.

The behavior of the nitro groups in dinitrobenzene is not without controversy. Dinitrobenzene substituent reactions have been studied before $[13,14]$. The spatial conformations of dinitrobenzene monomers are complex and are determined by several conjugation effects that can lead to misinterpretation of the data on the vibrational modes of dinitrobenzene [15]. This manuscript aims to further understand the differences among the three isomers of dinitrobenzene.

\section{Methods}

All the molecular modeling and single-point energy computations were performed using the Gaussian 09 package [16]. The twelve conjugated bonds in each isomer necessitated a model that can account for the delocalization of the charges in dinitrobenzene, so a model that considers intramolecular quantum interactions must be used to ensure precision. The B3LYP functional was used for the modeling, taking the required interactions into account, as well as being a standard for DFT computations. The basis set used was 6-31G(d), primarily because this basis set can be applied to larger molecules, unlike some of the more complex sets. Only qualitative agreement of the potential energy surface is needed with the physical parameters, because all that matters for the accurate determination of spatial conformations is that the torsional energy minima are in the correct places. Thus, relative differences in energy are significant, whereas the absolute determination of the energy surface is not necessary.

Many individual calculations can be combined to yield the torsional potential energy surface. To plot the surface, a $64 \times 64$ scan over the torsional angles of the nitro groups was performed for each isomer, allowing all parameters to vary except for the torsional angles of the two $\mathrm{NO}_{2}$ groups frozen during the scan. Thus, the resolution of the angle scans was $\pi / 32$ radians, the increment used for the scans. No symmetry restrictions were used during any of the computations given that torsional rotations break the molecular symmetry. The energy values at every point were tabulated using a script and then plotted as a function of the torsional angles of the two nitro groups. The symmetry of the potential energy surface was exploited so as to determine whole potential energy surface for each isomer from just one-quarter of the points. In (1), we present an expression for the potential energy surface obtained by expanding the potential in a leastsquares fit of Legendre polynomials. This expansion enables analytical insight into the potential energy surfaces of the three dinitrobenzene isomers, which is useful for comparison with previous theoretical and experimental results.

\section{Results}

The torsional PES of each isomer, expressed as a function of the torsional angles, can be expanded in terms of Legendre polynomials:

$$
\Phi\left(\theta_{1}, \theta_{2}\right)=b+c_{1} P_{1}\left(\cos \left(\theta_{1}+\varphi\right)\right)
$$




$$
\begin{aligned}
& +c_{2} P_{2}\left(\cos \left(\theta_{1}+\varphi\right)\right)+c_{3} P_{3}\left(\cos \left(\theta_{1}+\varphi\right)\right) \\
& +c_{4} P_{4}\left(\cos \left(\theta_{1}+\varphi\right)\right)+d_{1} P_{1}\left(\cos \left(\theta_{2}+\varphi\right)\right) \\
& +d_{2} P_{2}\left(\cos \left(\theta_{2}+\varphi\right)\right)+d_{3} P_{3}\left(\cos \left(\theta_{2}+\varphi\right)\right) \\
& +d_{4} P_{4}\left(\cos \left(\theta_{2}+\varphi\right)\right)+f_{1} P_{1}\left(\cos \left(\theta_{1}+\varphi\right)\right) \\
& \times P_{1}\left(\cos \left(\theta_{2}+\varphi\right)\right)+f_{2}\left(P_{1}\left(\cos \left(\theta_{1}+\varphi\right)\right)\right. \\
& \times P_{2}\left(\cos \left(\theta_{2}+\varphi\right)\right)+P_{2}\left(\cos \left(\theta_{1}+\varphi\right)\right) \\
& \left.\times P_{1}\left(\cos \left(\theta_{2}+\varphi\right)\right)\right)+f_{3}\left(P_{1}\left(\cos \left(\theta_{1}+\varphi\right)\right)\right. \\
& \times P_{3}\left(\cos \left(\theta_{2}+\varphi\right)\right)+P_{3}\left(\cos \left(\theta_{1}+\varphi\right)\right)
\end{aligned}
$$

$$
\begin{aligned}
& \left.\times P_{1}\left(\cos \left(\theta_{2}+\varphi\right)\right)\right)+f_{4} P_{2}\left(\cos \left(\theta_{1}+\varphi\right)\right) \\
& \times P_{2}\left(\cos \left(\theta_{2}+\varphi\right)\right) .
\end{aligned}
$$

The coefficients for the expansions of the potential energy (in units of $\mathrm{Ha}$ ) for each isomer are presented in Table 1. The phase shift, $\varphi$, included in the expansions is zero for all three isomers, as the potential energies are symmetric around $0^{\circ}$ torsional angles.

The Pearson correlation coefficient, $r$, was calculated in order to quantify the agreement between the computational models and the Legendre polynomial expansion. The following equation gives the method by which the Pearson correlation coefficients for each isomer were determined:

$$
r=\sum_{i=0}^{n_{x}} \sum_{j=0}^{n_{y}} \frac{\sum_{i=0}^{n_{x}} \sum_{j=0}^{n_{y}} n \psi\left(\theta_{i}, \theta_{j}\right) \Phi\left(\theta_{i}, \theta_{j}\right)-\sum_{i=0}^{n_{x}} \sum_{j=0}^{n_{y}} \psi\left(\theta_{i}, \theta_{j}\right) \sum_{i=0}^{n_{x}} \sum_{j=0}^{n_{y}} \Phi\left(\theta_{i}, \theta_{j}\right)}{\sqrt{n \sum_{i=0}^{n_{x}} \sum_{j=0}^{n_{y}} \psi\left(\theta_{i}, \theta_{j}\right)-\left(\sum_{i=0}^{n_{x}} \sum_{j=0}^{n_{y}} \psi\left(\theta_{i}, \theta_{j}\right)\right)^{2}} \sqrt{n \sum_{i=0}^{n_{x}} \sum_{j=0}^{n_{y}} \Phi\left(\theta_{i}, \theta_{j}\right)-\left(\sum_{i=0}^{n_{x}} \sum_{j=0}^{n_{y}} \Phi\left(\theta_{i}, \theta_{j}\right)\right)^{2}}} .
$$

In the above equation, $\psi$ is the computational data set, $\Phi$ is the Legendre polynomial expansion, $\theta_{i}, \theta_{j}$ are the angles corresponding to the ( $i$ th, $j$ th) point in the array, the double summations sum over every point in the array, and $n$ is the number of points in the array. Thus, the Pearson correlation coefficient between the expressions and the calculated PES is 0.9993 for the paraisomer and 0.9968 for the metaisomer when ignoring fourth- and higher-order Legendre polynomials. The orthoisomer PES data is compared with the expansion, using fourth-order polynomials, with $r=0.9577$. The only isomer where the difference between the computed energy surface and its expansion is visually discernible is the orthoisomer.

3.1. Paradinitrobenzene. We have constructed the torsional PES for the three dinitrobenzene isomers using firstprinciples methods. The surface is symmetric, with a global minimum corresponding to $(0,0)$, the planar configuration. This minimum is repeated four times on the graph, because torsional angles of 0 and $\pi$ are identical for the nitro groups. The plot is shifted so as to be centered around the global minimum. The potentials are seen to be described precisely by a first-order term only, showing that the potential energy depends on the cosines of the torsional angles. This is expected considering the fact that the energy changes are predominantly due to the torques on the conjugated bonds, as neither intramolecular attractions nor steric effects are present to hinder the rotation of the nitro groups. The minimum energy conformation is displayed in Figure 2.

The torsional potential energy surface of paradinitrobenzene is plotted in Figure 3.

As would be expected from the opposition of the $\mathrm{NO}_{2}$ groups, no effects of the electronic repulsion can be seen, since the molecule is completely planar in its lowest energy state. A slice of the potential corresponding to symmetric rotations, where both angles vary simultaneously, or in mathematical terms, $\Phi(\theta, \theta)$, is shown in Figure 4 .

The torsional energy is seen to have a behavior very similar to the cosine squared dependence on the angle that would be expected if no steric effects were present.

3.2. Metadinitrobenzene. The minimum energy conformation of metadinitrobenzene is displayed in Figure 5.

As for the paraisomer, the ground state conformation of the metaisomer is clearly planar. The torsional PES of metadinitrobenzene is plotted in Figure 6.

The global energy minimum of the surface is seen to be located at $(0,0)$. The plot with only one nitro group rotating is described, as before, by a first-order term, showing that the energy is related to the squared cosine of the torsional angle of the nitro group. Just as before, four identical energy minima are present, owing to the fourfold symmetry of the torsional PES. The characteristics of the torsional energy surface are seen to be similar to what is observed with the paraisomer, showing that the repulsive interactions between the $\mathrm{NO}_{2}$ groups have minimal effect on the geometry of the molecule. This conformation is also seen to be clearly planar. Thus, it is apparent that the repulsion between the $\mathrm{NO}_{2}$ groups is not straining the molecule. A slice of the potential, again for symmetric rotations, with both torsional angles rotated identically, is given in Figure 7 and is seen to be similar in all respects to the plot of the torsional potential of the paraisomer, showing that the steric repulsion is also very small in the metaisomer.

3.3. Orthodinitrobenzene. The torsional PES of orthodinitrobenzene, presented in Figure 8, is seen to be much different from the other two surfaces.

Here, eight minima are observed to be present in the surface. The global energy minima are not observed at $(0,0)$; 
TABLE 1: Coefficients of the Legendre polynomial expansion. For each expansion, we truncate the polynomials once data at the lower energies fit well. The terms marked with "*” are zero because of symmetries in the PES or are not used in the model because sufficient agreement was obtained with lower-order terms.

\begin{tabular}{lccc}
\hline Calculated coefficients $(\mathrm{mHa})$ & Paraisomer & Metaisomer & Orthoisomer \\
\hline$b$ & 9.8006 & 10.7201 & 10.0467 \\
$c_{1}$ & -5.6462 & -6.0068 & 0.0000 \\
$c_{2}$ & 0.2811 & 0.2856 & 4.8531 \\
$c_{3}$ & 0.0155 & 0.0136 & 0.0000 \\
$c_{4}$ & $0^{*}$ & $0^{*}$ & 2.1196 \\
$d_{1}$ & -5.6462 & -6.0068 & 0.000 \\
$d_{2}$ & 0.2811 & 0.2856 & 4.8531 \\
$d_{3}$ & 0.0155 & 0.0136 & 0.0000 \\
$d_{4}$ & $0^{*}$ & $0 *$ & 2.1196 \\
$f_{1}$ & 0.2850 & 0.0664 & 19.2731 \\
$f_{2}$ & $0^{*}$ & $0^{*}$ & 0.0000 \\
$f_{3}$ & $0^{*}$ & $0^{*}$ & 1.0792 \\
$f_{4}$ & $0^{*}$ & $0^{*}$ & 18.4973 \\
\hline
\end{tabular}

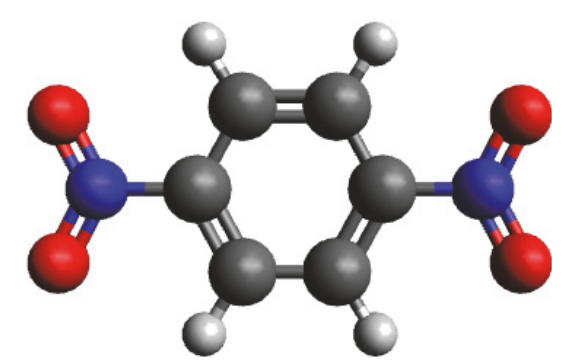

FigurE 2: The ground state conformation of paradinitrobenzene.

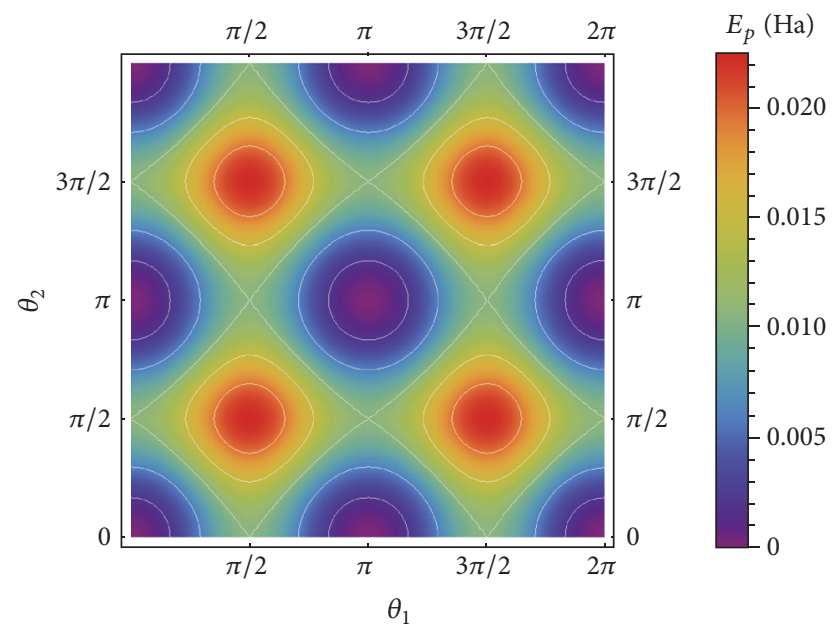

FIGURE 3: Torsional PES of paradinitrobenzene. The conformational energies (in Hartree) of the minima are zero, while the barriers around $(0, \pi / 2)$ are $0.0107 \mathrm{Ha}$ and $0.0225 \mathrm{Ha}$ at $(\pi / 2, \pi / 2)$.

rather, the minima are seen at $(\pi / 4,5 \pi / 16)$ and the seven points symmetric to it. A complex relation between the potential energy of the molecule and the rotations of the nitro groups is observed. This complicated dependence of the potential energy upon the torsion angles of the nitro

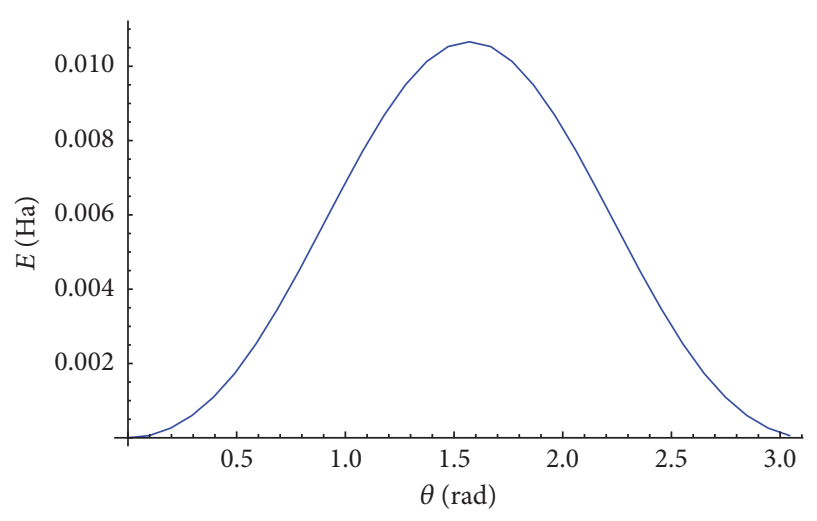

FIgUre 4: Plot of the electronic energy above the minimum for the paraisomer as a function of the torsional angle of the $\mathrm{NO}_{2}$ groups, using the computed Legendre polynomial expansion.

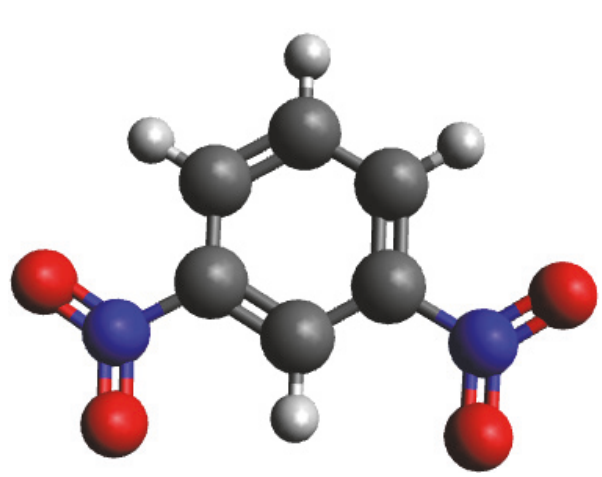

FIGURE 5: The ground state conformation of metadinitrobenzene.

groups results from the multiplicity of factors affecting their rotations. These factors include steric repulsion between the $\mathrm{NO}_{2}$ groups, the formation of conjugated ions attracting the $\mathrm{H}$ atoms located at adjacent positions on the benzene ring, the resistance of the conjugated $\pi$ orbitals to the rotation 


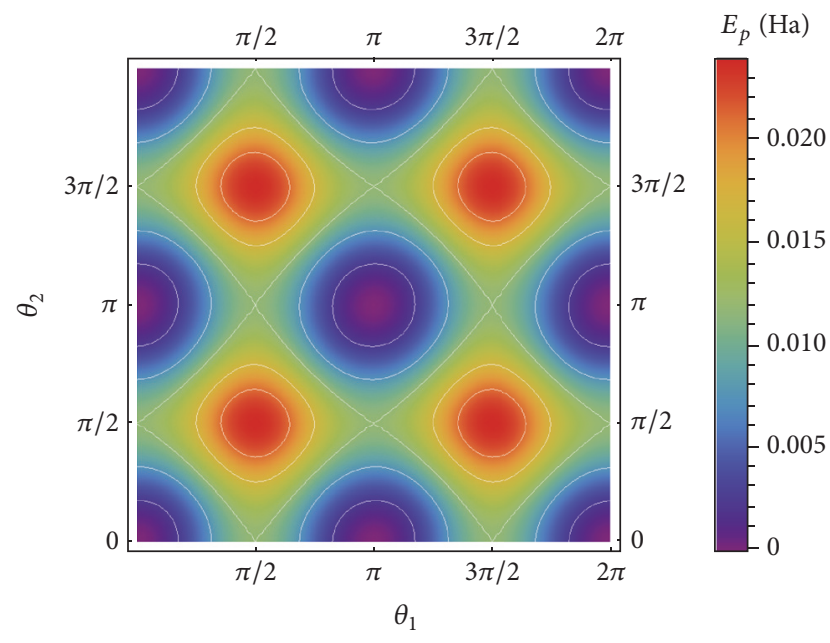

FIGURE 6: The torsional PES of metadinitrobenzene. The conformational energies (in Hartree) of the minima are zero, while the barriers around $(0, \pi / 2)$ are 0.0118 and 0.0239 at $(\pi / 2, \pi / 2)$.

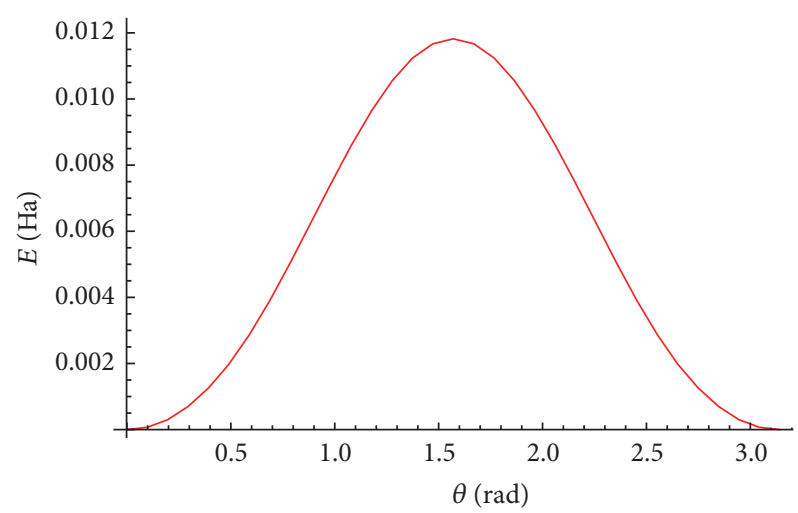

FIgURE 7: The Legendre expanded electrostatic energy above the minimum as a function of the C-N bond angles is plotted for the metaisomer.

of the $\mathrm{NO}_{2}$ groups, and the stress on the $\mathrm{C}-\mathrm{N}$ bond caused by the repulsion of the $\mathrm{NO}_{2}$ groups. From the graphic of the minimum energy conformation of orthodinitrobenzene, shown in Figure 9, it is clearly evident that the $\mathrm{NO}_{2}$ groups are not in the plane of the benzene ring.

It can also be seen that each of the C-N bonds is bent away from the other $\mathrm{NO}_{2}$ group, increasing the spacing of the oxygen atoms from each other. The torsional stresses are seen to be great enough to force the molecule out of the planar conformation preferred by bond hybridization, because the repulsion between the $\mathrm{NO}_{2}$ groups is very strong. Thus, the orthoisomer is the only one of the three isomers studied which is greatly influenced by steric repulsion. The slice of the torsional PES of the orthoisomer pertaining to symmetric rotations is illustrated in Figure 10.

To verify that the strain on the orthoisomer was not improperly considered by the constraints of the scan, all the bond length and angle constraints were relaxed for the conformation associated with the global energy minimum, and the strains on each atom were minimized. Table 2 indicates

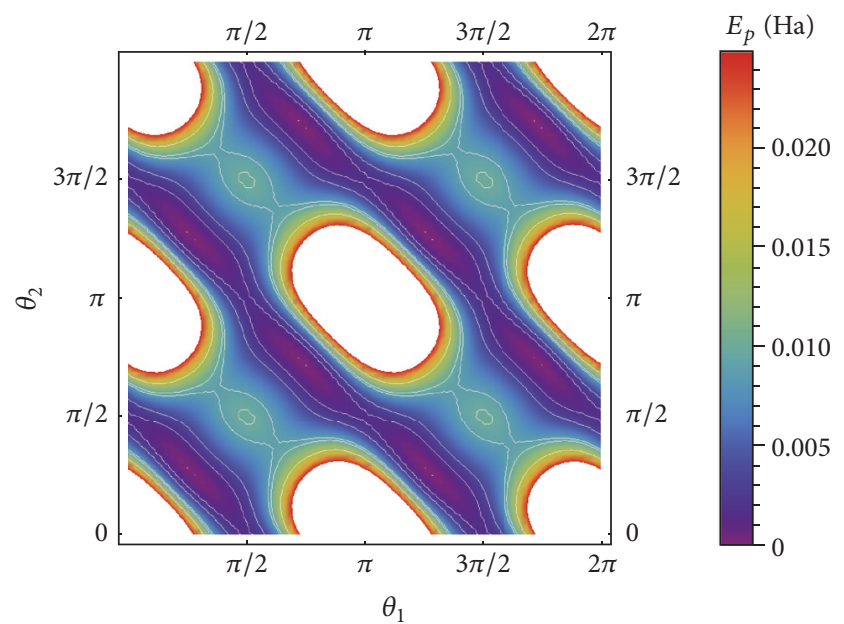

FIgURE 8: Torsional PES of orthodinitrobenzene. The conformational energies (in Hartree) of the minima are set to zero. The barriers around $(\pi, \pi)$ are $0.1989 \mathrm{Ha}$, and the barriers are $0.0101 \mathrm{Ha}$ at $(\pi / 2, \pi / 2)$. Different from the previous two isomers, the zero energy point is located at $(\pi / 4,5 \pi / 16)$ and the symmetric permutations. The Legendre expansion places the minimum at $(1.107,1.107)$, which is closest to the point $(\pi / 4, \pi / 4)$.

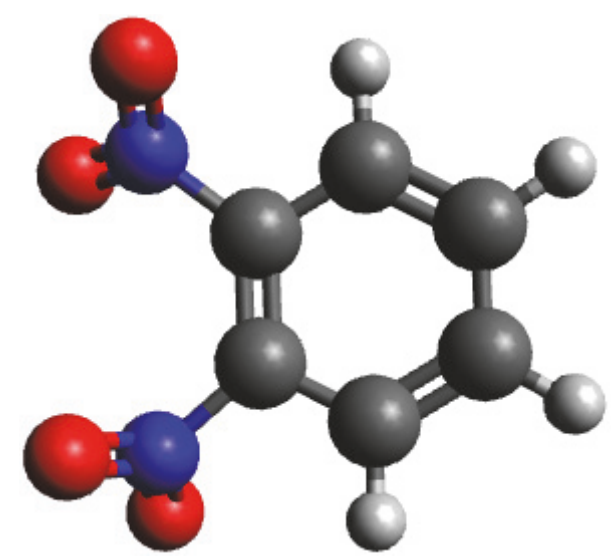

FIGURE 9: The lowest energy conformation of orthodinitrobenzene.

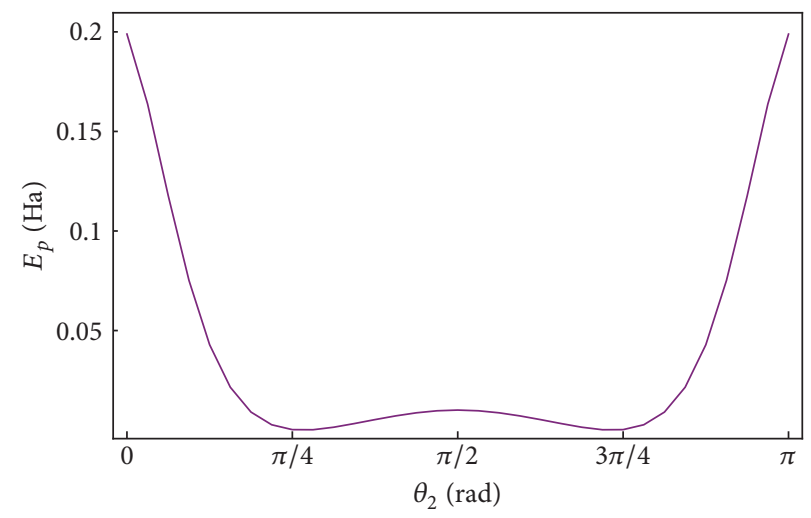

FIgURE 10: A slice of the Legendre polynomial expansion of orthodinitrobenzene, considering symmetric rotations of the nitro groups, with both nitro groups rotating together. 
TABLE 2: The effect of relaxing the scan constraints at the orthodinitrobenzene minimum energy conformation.

\begin{tabular}{lcc}
\hline Orthodinitrobenzene bonds & Average original length $(\AA)$ & Average final length $(\AA)$ \\
\hline C-C & 1.395 & 1.391 \\
C-H & 1.085 & 1.086 \\
C-N & 1.475 & 1.453 \\
N-O & 1.227 & 1.226 \\
\hline
\end{tabular}

the changes in bond lengths obtained by the minimization. None of the angles deviated significantly from the values computed by the scan.

\section{Conclusion}

We have constructed the torsional PES for dinitrobenzene isomers using first-principles methods. The paraisomer and metaisomer are found to have weak interactions between nitro groups, so the torsional potentials can be accurately modeled using very few terms in an expansion. For the orthoisomer, however, the strong steric repulsion between the adjacent $\mathrm{NO}_{2}$ groups makes the theoretical form of the potential much more complex. However, the fourth-order expansion for the potentials only differs from the computed potentials towards the energy maximum. This difference is not important as regards the goal of this research, which is to enable the computation of lowest energy structures of molecules. Since the goal is to determine the location in angle space of the energy minimum, the exact characteristics of an energy maximum have no relevance to the usefulness of the potential. The result is the confirmation that the $\mathrm{NO}_{2}$ groups of orthodinitrobenzene do not lie in the plane of the benzene ring. A previous report stated that the $\mathrm{NO}_{2}$ groups are not planar for the orthoisomer [17] but this has been corrected by Tomkinson [18]. It may be noted that the symmetry properties used in that computational study are the cause of this discrepancy. Certainly, the potential energy surface of orthodinitrobenzene is the most complex because of the preponderance of hindrances to the rotations of the $\mathrm{NO}_{2}$ groups.

The modeling of the properties of two-rotor molecules, an important first step to allow the reduction of the problem of a molecular geometry to a system of linear differential equations, can be accomplished to great accuracy by the computation of torsional potentials. This method can be tailored to available computational resources by adjusting the spacing between the points in the torsional potential, as well as by altering the region around the torsional minima for which the potential will be computed. The set of correlations between combinations of two-rotor systems in a molecule can be computed far more easily than a normal geometry optimization, because the number of molecular degrees of freedom is limited to two at a time.

\section{Disclosure}

Some of the results in this article were presented at the 2015 APS March Meeting [19].

\section{Conflicts of Interest}

The authors declare that they have no conflicts of interest.

\section{Acknowledgments}

This work used the OSU High Performance Computing Center at Oklahoma State University supported in part through the National Science Foundation (Grant no. OCI1126330). The authors thank S. Dai, A. Adams, and C. Fennell for useful discussions.

\section{References}

[1] L. Bertini, C. Greco, L. De Gioia, and P. Fantucci, "DFT/TDDFT exploration of the potential energy surfaces of the ground state and excited states of $\mathrm{Fe} 2(\mathrm{~S} 2 \mathrm{C} 3 \mathrm{H} 6)(\mathrm{CO}) 6$ : A simple functional model of the [FeFe] hydrogenase active site," Journal of Physical Chemistry A, vol. 113, no. 19, pp. 5657-5670, 2009.

[2] H.-H. G. Tsai, H.-L. S. Sun, and C.-J. Tan, "TD-DFT study of the excited-state potential energy surfaces of 2-(2IHydroxyphenyl)benzimidazole and its amino derivatives," The Journal of Physical Chemistry A, vol. 114, no. 12, pp. 4065-4079, 2010.

[3] C. Møller and M. S. Plesset, "Note on an approximation treatment for many-electron systems," Physical Review, vol. 46, no. 7, pp. 618-622, 1934.

[4] D. R. Hartree, "The Wave Mechanics of an Atom with a NonCoulomb Central Field Part I Theory and Methods," Mathematical Proceedings of the Cambridge Philosophical Society, vol. 24, no. 1, pp. 89-110, 1928.

[5] V. Fock, "Näherungsmethode zur Lösung des quantenmechanischen Mehrkörperproblems," Zeitschrift für Physik, vol. 61, no. 1-2, pp. 126-148, 1930.

[6] A. D. Becke, "A new mixing of Hartree-Fock and local densityfunctional theories," The Journal of Chemical Physics, vol. 98, no. 2, pp. 1372-1377, 1993.

[7] W. Kohn, A. D. Becke, and R. G. Parr, "Density functional theory of electronic structure," The Journal of Physical Chemistry, vol. 100, no. 31, pp. 12974-12980, 1996.

[8] A. Bongini and A. A. Bottoni, "A theoretical investigation of the torsional potential in 3,3'-dimethyl-2,2'-bithiophene and 3,4' Dimethyl-2,2'-bithiophene: a comparison between HF, MP2, and DFT theory," The Journal of Physical Chemistry A, vol. 103, no. 34, pp. 6800-6804, 1999.

[9] S. Bell, P. Groner, G. A. Guirgis, and J. R. Durig, "FarInfrared Spectrum, ab Initio, and DFT Calculations and TwoDimensional Torsional Potential Function of Dimethylallene (3-Methyl-1,2-butadiene)," The Journal of Physical Chemistry A, vol. 104, no. 3, pp. 514-520, 2000. 
[10] G. Cinacchi and G. Prampolini, "DFT study of the torsional potential in ethylbenzene and ethoxybenzene: The smallest prototypes of alkyl- and alkoxy-aryl mesogens," Journal of Physical Chemistry A, vol. 107, no. 26, pp. 5228-5232, 2003.

[11] M. D. Hanwell, D. E. Curtis, D. C. Lonie, T. Vandermeerschd, E. Zurek, and G. R. Hutchison, "Avogadro: an advanced semantic chemical editor, visualization, and analysis platform," Journal of Cheminformatics, vol. 4, no. 1, article 17, 2012.

[12] J. H. Freed, P. H. Rieger, and G. K. Fraenkel, "Alternating linewidths in the ESR spectra of dinitrobenzene anion radicals," The Journal of Chemical Physics, vol. 37, no. 8, pp. 1881-1882, 1962.

[13] M. Polášek, F. Tureček, P. Gerbaux, and R. Flammang, "Nitrobenzene Isomers," The Journal of Physical Chemistry A, vol. 105, no. 6, pp. 995-1010, 2001.

[14] V. Krishnakumar and N. Prabavathi, "Analysis of vibrational spectra of 1-chloro-2,4-dinitrobenzene based on density functional theory calculations," Spectrochimica Acta - Part A: Molecular and Biomolecular Spectroscopy, vol. 72, no. 4, pp. 738-742, 2009.

[15] J. Clarkson and W. E. Smith, "A DFT analysis of the vibrational spectra of nitrobenzene," Journal of Molecular Structure, vol. 655, no. 3, pp. 413-422, 2003.

[16] M. Frisch, G. Trucks, H. B. Schlegel et al., Gaussian 09, revision A. 02; Gaussian, Inc, vol. 19, pp. 227-238, 2009.

[17] D. Mahadevan, S. Periandy, and S. Ramalingam, "Comparative vibrational analysis of 1,2-Dinitro Benzene and 1-Fluoro-3Nitro Benzene: a combined experimental (FT-IR and FTRaman) and theoretical study (DFT/B3LYP/B3PW91)," Spectrochimica Acta - Part A, vol. 84, no. 1, pp. 86-98, 2011.

[18] J. Tomkinson, "Comment on: "comparative vibrational analysis of 1,2-dinitrobenzene and 1-fluoro-3-nitrobenzene" by S Ramalingam, S. Periandy et al. [Spectrochim. Acta A 84 (2011) 8698]," Spectrochimica Acta - Part A: Molecular and Biomolecular Spectroscopy, vol. 91, no. 6, pp. 431-432, 2012.

[19] P. Smith and M. Borunda, "Determination of the Torsional Potential of Energy Surfaces of the Ortho- Meta-, and ParaIsomers of Dinitrobenzene," in Proceedings of the APS Meeting Abstracts, 2015. 

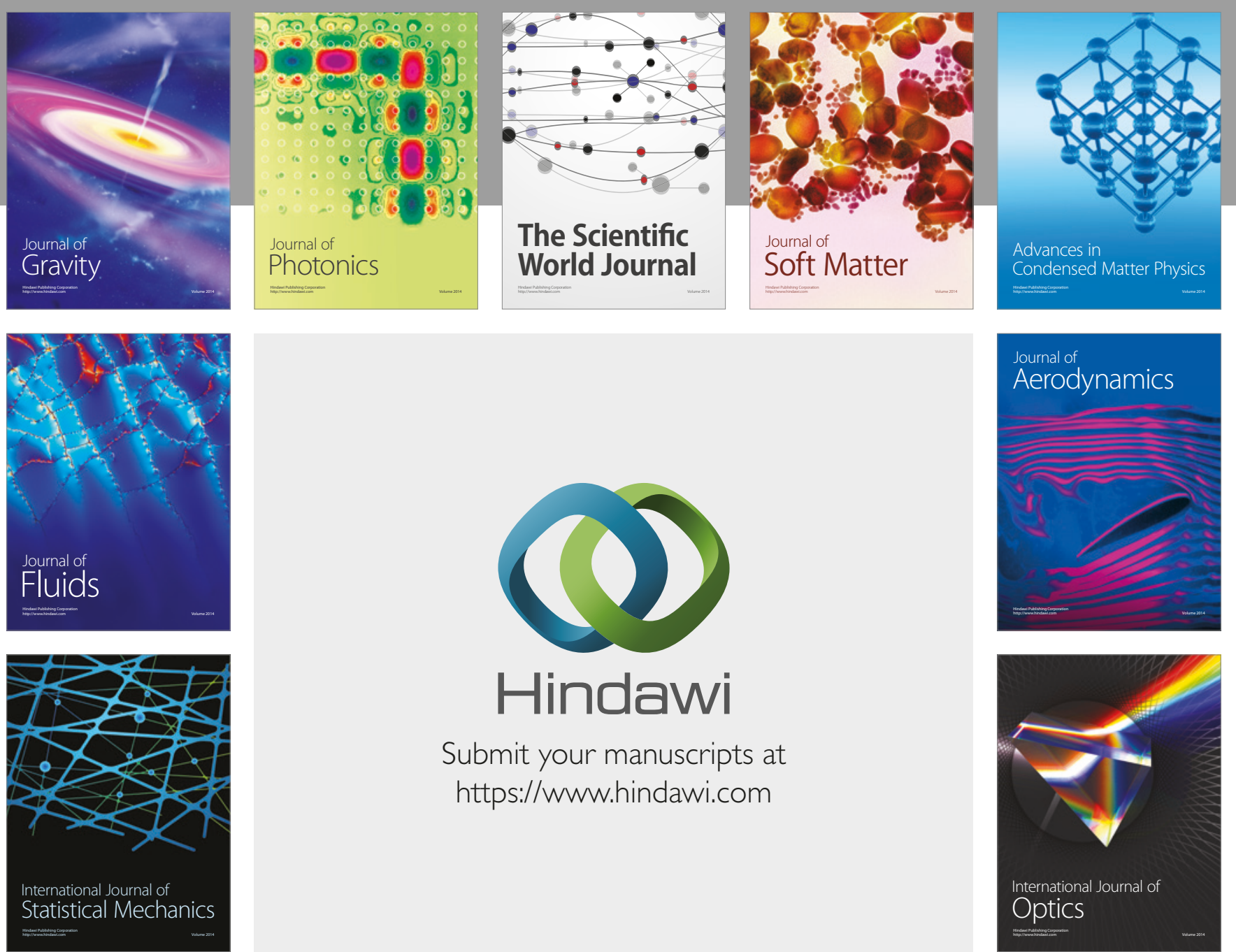

Submit your manuscripts at

https://www.hindawi.com
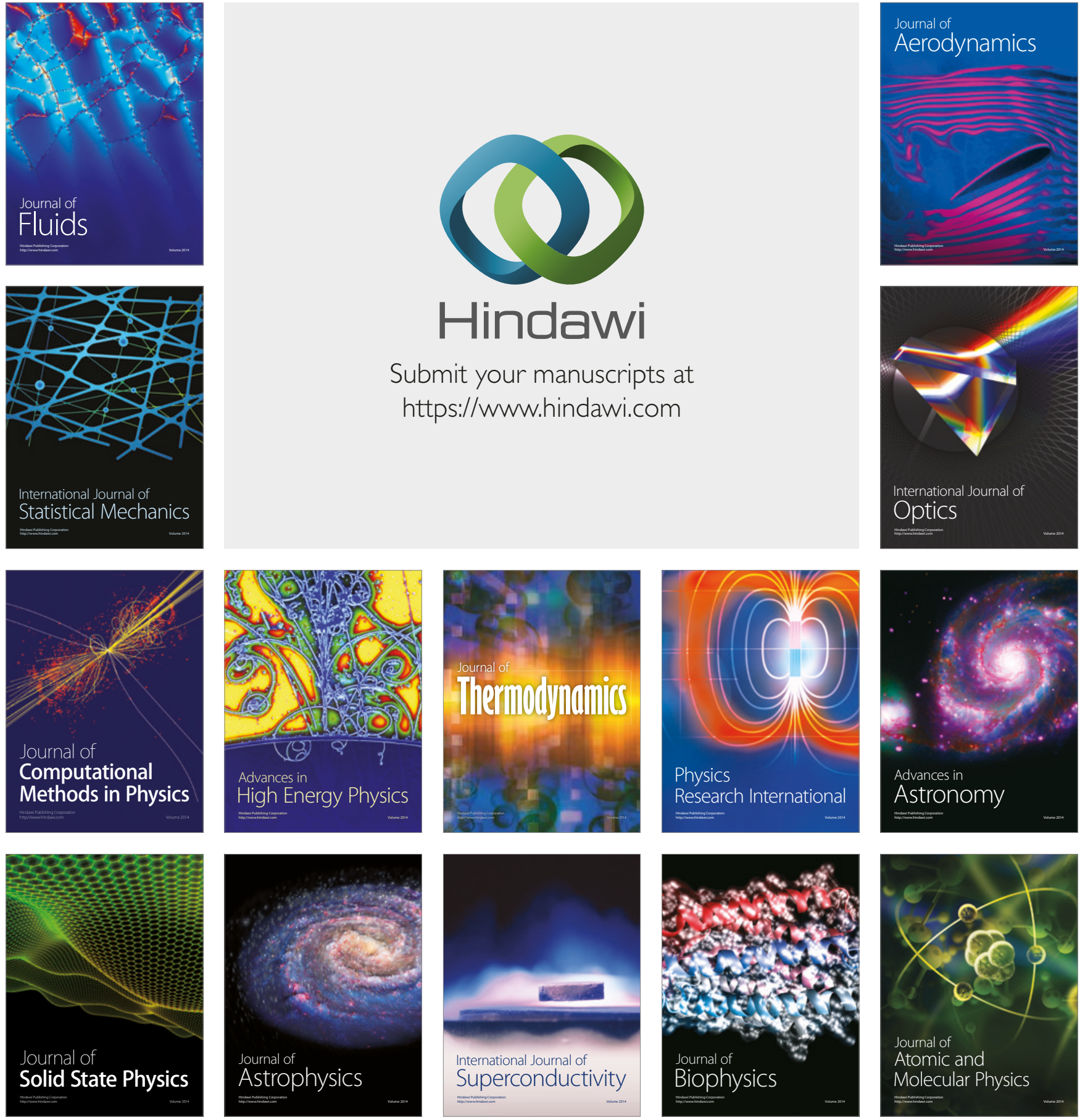